\title{
Aero-thermal flow characterization downstream of an NGV cascade by five-hole probe and filtered Rayleigh scattering measurements
}

\author{
Ulrich Doll • Michael Dues • Tommaso Bacci • Alessio Picchi • Guido \\ Stockhausen • Christian Willert
}

Received: date / Accepted: date

\begin{abstract}
The characterization of pressure, temperature and velocity fields in turbomachinery flows typically relies on well-proven probe-based technology such as pneumatic probes, hot-wire sensors or thermocouples. These devices have to be introduced into narrow flow channels and by that obstruct part of the duct at the actual measuring position, which can significantly alter the aerodynamic performance of the components under investigation. In this contribution, measurement results of a commercially available five-hole probe with mounted temperature sensor and laser-optical filtered Rayleigh scattering diagnostics are acquired downstream of a nozzle guide vane cascade with lean-burn combustion representative inlet flow distortions. Pressure results obtained by both methods are found to be on a
\end{abstract}

\section{U. Doll}

Paul Scherrer Institute (PSI), Laboratory of Thermal Processes and Combustion, 5232 Villigen PSI, Switzerland

Tel.: +41563102287

E-mail: ulrich.doll@psi.ch

\section{Dues}

ILA R\&D GmbH, Karl-Heinz-Beckurts Str. 13, 52428 Juelich, Germany

T. Bacci

Dipartimento di Ingegneria industriale, University of Florence, 50139 Florence, Italy

\section{A. Picchi}

Dipartimento di Ingegneria industriale, University of Florence, 50139 Florence, Italy

\section{G. Stockhausen}

German Aerospace Center (DLR), Institute of Propulsion Technology, Measurement Technology, Linder Hoehe, 51147 Cologne, Germany

C. Willert

German Aerospace Center (DLR), Institute of Propulsion Technology, Measurement Technology, Linder Hoehe, 51147 Cologne, Germany similar absolute level. However, the unavoidable movement of optical elements following the facilities heatup procedure prevents a sufficient sensitivity of pressure data obtained by filtered Rayleigh scattering and therefore an identification of certain flow structures related to probe interference on the static pressure field. Concerning temperature data, results of both methods, despite of the probe data being shifted in lateral direction, are found to be in good agreement in topology as well as absolute level. The strongest intrusive influence of the probe body as well as a bias to probe readings in certain flow regions is identified when comparing velocity data by means of the optical Doppler frequencyshift. Whereas filtered Rayleigh scattering results follow a smooth and continuous trend, five-hole probe data is characterized by steep gradients inside the airfoil wakes as well as differing slopes in the flow passages. These findings are backed by a comparison of five-hole probe velocities with a CFD solution, revealing an erroneous reading of axial velocities inside the wake areas. The study concludes that the presented filtered Rayleigh scattering diagnostics, with estimated accuracies of $50-90 \mathrm{hPa}, 2-3 \mathrm{~K}$ and $2-3 \mathrm{MHz}$ in pressure, temperature and Doppler frequency-shift, is a viable alternative to conventional probe based measuring approaches in characterizing the aero-thermal properties of turbomachinery flows.

Keywords five-hole probe $\cdot$ filtered Rayleigh scattering · laser diagnostics · pressure · temperature · flow velocity $\cdot$ planar measurement $\cdot$ turbomachinery

PACS 42.62.Fi · 47.80.Fg - 47.80.Jk · 33.20.Fb · 42.25.Bs $\cdot 07.20 . \mathrm{Dt} \cdot 06.30 . \mathrm{Gv} \cdot 07.07 . \mathrm{Df}$ 


\section{Introduction}

The introduction of lean burn combustion concepts is considered a promising path on the route of increasing the overall efficiency of aero-engines while at the same time meeting tightened emission targets (von der Bank et al, 2014). Novel fuel injector designs are required, which eventually lead to a substantial redirection of air mass inside the core engine. As a consequence, highly swirling flows, characterized by severe temperature gradients (hot streaks) and significant turbulence approach the turbine. These unsteady flow fields significantly challenge the reliability of common design procedures (von der Bank et al, 2014; Chevrier and Bertrand, 2017).

Within this framework, a three-sector rig made up of a combustor simulator and a nozzle guide vane (NGV) cascade was installed at THT Lab (Laboratory of Technologies for High Temperature) of the University of Florence. In the combustor simulator, representative temperature distortions, swirl and turbulence fields are generated through the mixing of a heated main flow passing three axial swirlers and liner coolant flows at ambient temperature (Bacci et al, 2015). The focus of the present study lies on the experimental characterization of pressure, temperature and velocity distributions downstream of the NGV cascade.

The experimental characterization of aero-thermal flow properties in such configurations typically relies on well-proven probe based technology such as pneumatic multi-hole devices, hot-wire probes or thermocouples (see e. g. Heinke et al (2004); Qureshi et al (2012); Luque et al (2015)). Due to their robustness and reliability, pneumatic multi-hole probes are widely used in applications related to turbomachinery. Nevertheless, it is well known that, when exposed to strong gradients fields, significant measurement error due to the spatial placement of the pressure taps on the probe geometry is likely to be introduced (Hoenen et al, 2012; Aschenbruck et al, 2015; Sanders et al, 2017). In this respect, Hoenen et al (2012) carried out a conclusive study on the detrimental effects on measurement accuracies when performing five-hole probe measurements in the wake region of turbine blade airfoils. The authors also present a correction method, which has to be included in the calibration procedure. In addition to issues regarding the measurement principle, an interaction between probe head and airfoil wake flow was found in Aschenbruck et al (2015). In a numerical study carried out by Sanders et al (2017) it was shown that effects of partially blocking the flow downstream of a stator row by introducing a pressure probe are recognizable upstream of the cascade as well as in neighboring passages.
Considering the above mentioned aspects in the context of using probes in turbomachinery environments, due to their non-invasive nature, laser-optical diagnostics appear to be the natural choice for these kinds of applications. Regarding planar methods, in particular particle image velocimetry (PIV) was successfully applied in numerous studies, ranging from the characterization of stator passages and wakes (Bryanston-Cross et al, 1992; Woisetschläger et al, 2003) to applications in rotating machinery (Wernet, 2000; Voges et al, 2012). As an alternative approach to PIV, Doppler global velocimetry with frequency modulation (FM-DGV) was applied on a transonic cascade (Fischer et al, 2012) as well as a rotating turbine stage (Fischer et al, 2013).

Despite their general ability to deliver time-resolved three-component velocity fields in turbomachinery environments, both PIV and DGV suffer from their susceptibility to laser scattering from surfaces, so that affected image areas have to be masked and thus cannot be included in the data evaluation (Woisetschläger et al, 2003; Voges et al, 2012; Fischer et al, 2013). In addition, seeding particles tend to deposit on windows, which, due to the degrading quality of optical access, in consequence leads to reduced testing periods. In this regard the filtered Rayleigh scattering technique (FRS) (Miles and Lempert, 1990) may prove a valuable alternative to existing approaches. Extended by the method of frequency scanning (FSM-FRS) (Forkey, 1996; Doll et al, 2014), the technique is capable to acquire timeaveraged pressure, temperature and velocity maps simultaneously. FRS relies on molecular scattering only, no seeding particles have to be added to the flow. In utilizing molecular absorption bands in the range of the excitation wavelength, laser stray light from surfaces can be effectively attenuated, yielding experimental results in the direct vicinity of highly luminous surfaces. The technique was already employed on several turbomachinery related applications, including multi-parameter measurements in air flows (Doll et al, 2014) as well as in combustion environments (Schroll et al, 2017; Doll et al, 2017a).

In this contribution, aero-thermal flow properties behind the NGV cascade of a three-sector gas turbine combustor simulator will be investigated by means of a commercially available five-hole probe with mounted temperature sensor as well as laser-optical FSM-FRS diagnostics. Present limitations of the FSM-FRS technique with respect to its applicability under flow conditions experienced in the current turbomachinery setup as well as resulting measurement accuracies in the measured quantities will be examined. Based on a detailed analysis of the differences between results obtained with both methods, the probe's intrusive effect, issues con- 


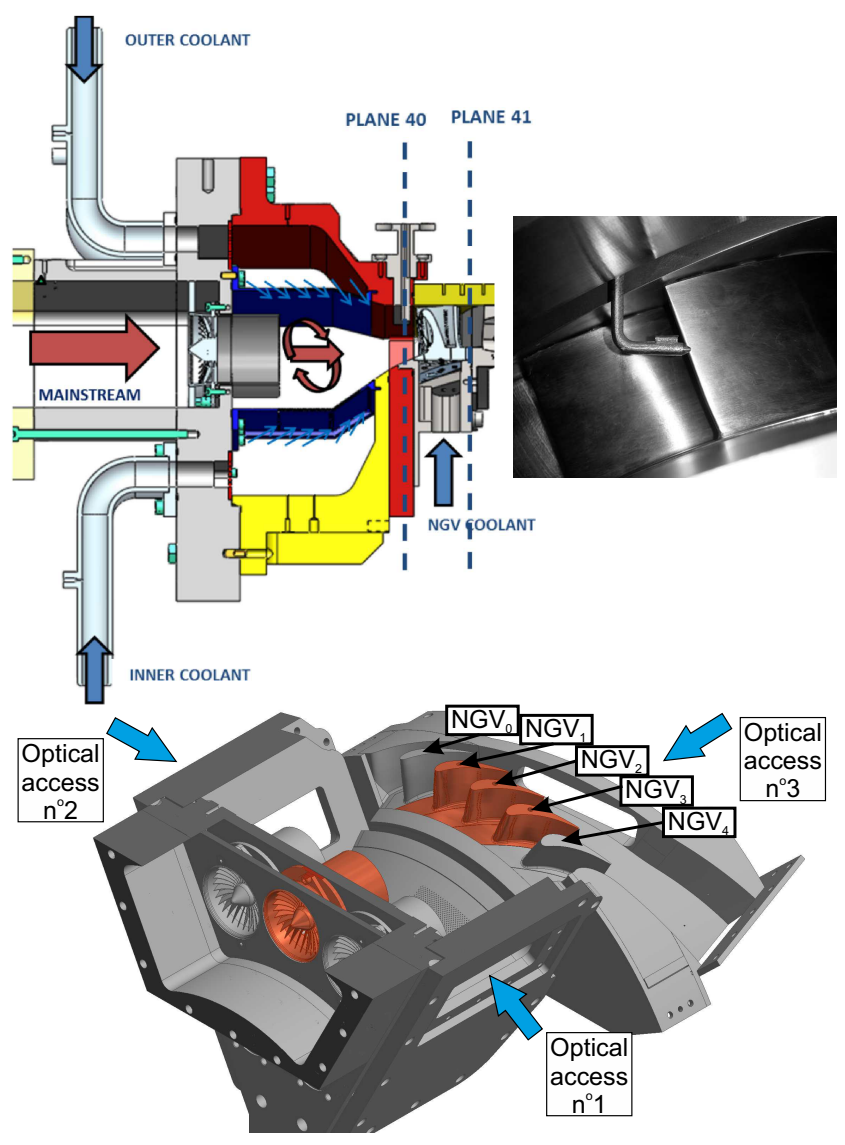

Fig. 1 (Top) Schematic view of the test rig with main stream and coolant mass flows highlighted in red and blue. The photograph shows the probe's positioning inside the test section. (Bottom) Sectional view of the test rig's CAD model. The central sector is coloured in orange, blue arrows indicate optical access to the rig.

cerning the probe's positioning as well as the impact of particular flow phenomena on the probe's measurement accuracy will be discussed.

\section{Experimental facilities}

\subsection{Three-sector combustor simulator}

The experimental survey was carried out on a nonreactive, three-sector combustor simulator rig coupled with a high pressure NGV cascade. According to Fig. 1, top, the pre-heated main stream passes through three axial swirlers, which create a highly swirling flow in the chamber. Two separate lines at ambient temperature feed the effusion cooled inner and outer liner. The interaction between the three flows generates a lean burn combustor representative flow field at the combustor exit. Relevant temperature distortions, together with high degree of swirl $\left( \pm 50^{\circ}\right)$ and turbulence intensity (up to $\sim 30 \%$ ) are achieved in Plane 40, nominally
Table 1 Operating points

\begin{tabular}{cccc}
\hline & $\begin{array}{c}\text { chamber } \\
\text { pressure }(\mathrm{hPa})\end{array}$ & $\begin{array}{c}\text { pre-heat } \\
\text { temp. }\left({ }^{\circ} \mathrm{C}\right)\end{array}$ & $\begin{array}{c}\text { Average } \\
\text { Ma }(-)\end{array}$ \\
\hline Iso & 1270 & 27 (Ambient) & 0.7 \\
OP150 & 1480 & 150 & 0.64 \\
OP250 & 1480 & 250 & 0.77 \\
\hline
\end{tabular}

the combustor-turbine interface plane. The combustor simulator presents typical features of modern lean burn combustors in the form of main-coolant mass flow split $(65 \%-35 \%)$, effusion cooled liners without dilution holes, and limited axial dimensions $(\sim 2.5$ swirler diameters). The three sector configuration was chosen in order to make the central sector flow field, which is the target of measurements, insensitive to the presence of the rig lateral walls. Ducts of $55 \mathrm{~mm}$ length $(35 \%$ of the chamber length) are installed on the swirlers in order to postpone the swirling structure expansion and its interaction with liner cooling flows. Therefore enhanced temperature and flow distortions, representative for lean burn injection technology, can be achieved at the chamber exit (Bacci et al, 2015).

The NGV cascade visible in Fig. 1, bottom is made up of five vanes - six passages, in order to reach a swirler-to-vane count ratio of $1: 2$. The central vane has the leading edge aligned with the central swirler. Since only the central sector (i.e. two vane pitches) is subject of the investigation, only the three central airfoils $\left(\mathrm{NGV}_{1-3}\right)$ are film-cooled. Two dummy airfoils $\left(\mathrm{NGV}_{0}\right.$ and $\mathrm{NGV}_{4}$ ) have been installed at their sides. The NGV coolant flow is provided by a further line at ambient temperature. A back-pressure valve is used to regulate the rig pressurization. Operating points (OPs) investigated in this study and average Mach numbers reached at Plane 41 are summarized in Tab. 1.

The test rig is provided with different kinds of access to support the adopted measurement techniques. Two adaptive flanges are located upstream and downstream of the cascade (Plane 40 and Plane 41), in order to carry out probe traversing in these planes. Plane 40 is located half an axial chord upstream of the vane leading edge, while Plane 41 is about 0.25 axial chords $(\sim 9 \mathrm{~mm})$ downstream of the vane trailing edge. The upstream flange is easily recognizable in Fig. 1, top. The rig is also provided with three optical accesses (Fig. 1, bottom) to be exploited for optical measurements upstream and downstream of the vane profiles. The third access was used in the present investigation, in order to observe Plane 41 from downstream direction 


\subsection{Five-hole probe setup}

Probe measurements have been carried out by means of a Vectoflow ${ }^{\circledR}$ five-hole probe with $3 \mathrm{~mm}$ head size. A J-type thermocouple is installed on top of the probe shaft, which is approximately $4 \mathrm{~mm}$ from the pressure tap positions. Since a probe preswirl of $62.5^{\circ}$ was used in order to reduce the measured angles and increase the probe's accuracy, this results in an axial displacement of less than $2 \mathrm{~mm}$. The thermocouple is placed inside a shroud, as is common for Kiel probes, in order to make the thermocouple recovery factor more insensitive to high flow angles and reduce measurement uncertainty.

A traverse system installed on the downstream flange was used to automatically move the probe within Plane 41 , resulting in a measurement grid of 335 points. Radial and tangential resolutions of about $2.5 \mathrm{~mm}$ and $1^{\circ}$ were achieved, resulting in about 10 points per NGV pitch. Due the probe dimensions it was not possible to investigate the annulus areas in the very proximity of the channel boundaries: the investigated domain covers from 7 to $82 \%$ of the radial span.

For each mesh point data were acquired and averaged over a period of 2 seconds. In order to characterize the probe recovery factor, a thorough calibration procedure was carried out; yaw angles of up to $\pm 75^{\circ}$ and pitch angles of up to $+75^{\circ} /-60^{\circ}$, where investigated, while Mach numbers were increased up to 0.9. Within the measured range of Mach numbers and flow angles, pressure and temperature measurement uncertainty stays below $20 \mathrm{hPa}$ and $2 \mathrm{~K}$ of the respective reading. Resulting velocity uncertainties are estimated to $\sim 1.6 \%$ on a relative scale.

\subsection{FSM-FRS diagnostics}

The FRS method relies on the spectral discrimination between elastic laser light scattering from gas molecules, which holds information on density, pressure, temperature and flow velocity (through the optical Doppler frequency-shift) inside a probe volume and laser stray light from surfaces or large particles (Mie scattering) by means of molecular absorption. The underlying principle of the FRS technique is illustrated in Fig. 2. Surface or particle scattering has the same narrow bandwidth as the illuminating light source, while, due to the molecular motion, light scattered from gas molecules is spectrally broadened. In utilizing a molecular absorption line as notch filter, laser stray light can be strongly attenuated, while portions of Rayleigh scattering pass through the filter and form the FRS signal (Miles et al, 2001).

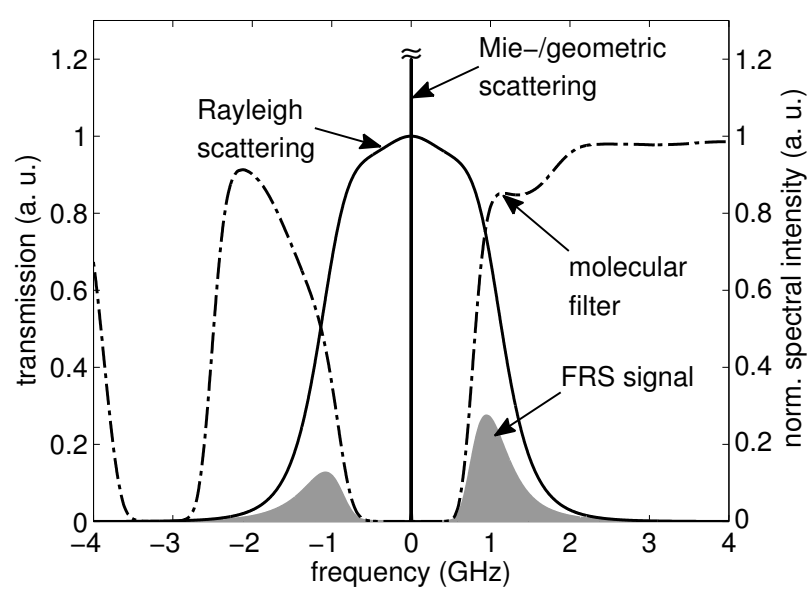

Fig. 2 Narrow bandwidth laser light scattering (Mie/geometric) is attenuated by the molecular filter (dashed-dotted), while portions of the spectrally broadened Rayleigh scattering pass through and form the FRS-signal.

The FSM-FRS signal intensity scattered from a small volume filled with gas molecules of a single species, which is accumulated by the detector at sensor element $i j$, can be described as (Forkey, 1996; Doll et al, 2014)

$$
\begin{array}{r}
S_{i j k}\left(\nu_{0, k}, p_{i j}, T_{i j}, \Delta \nu_{i j}, \Theta_{i j}\right)= \\
I_{0} n_{i j} R_{i j} \int_{-\infty}^{\infty} r_{i j}\left(\nu_{0, k}, p_{i j}, T_{i j}, \Theta_{i j}\right) \tau\left(\nu+\Delta \nu_{i j}\right) \mathrm{d} \nu,
\end{array}
$$

with $I_{0}$ as incident laser intensity, $n$ as number density and $R$ as the optical setup's efficiency. The integral denotes the convolution between the Rayleigh scattering's spectral lineshape $r$, which is a function of excitation frequency $\nu_{0}$, pressure $p$, temperature $T$, scattering angle $\Theta$ and the molecular filter's transmission curve $\tau$, which incorporates the Doppler frequency-shift $\Delta \nu$. As in FSM-FRS the laser's output frequency is scanned in discrete steps along the molecular filter's transmission curve, the subscript $k$ denotes the actual frequency value.

In order to use Eq. (1) to evaluate FSM-FRS data, it has to be assured, that between a reference measurement with pressure, temperature known and zero flow velocity and the actual experiments, the optical efficiency $R$ may not be varied (Doll et al, 2016). This cannot be ensured in the current setup, as the test section as well as it's tubing are expanding during the heat-up procedure. Thus, the modified model equation $Q_{i j k}=\frac{S_{i j k}}{\left\langle S_{i j}\right\rangle}$

introduced in Doll et al (2016) has to be used in the data evaluation procedure. In dividing each measured intensity $S_{i j k}$ by it's ensemble average over all frequencies $\left\langle S_{i j}\right\rangle$, the expression becomes independent of $R$, 
but suffers from a lower sensitivity to pressure. To further increase measurement accuracies, a correction method to account for laser-induced background scattering as well as a calibrated analytical formulation to model the Rayleigh scattering's spectral lineshape, both introduced in Doll et al (2016), are applied here.

Fig. 3 gives an overview of the FSM-FRS optical setup. The system relies on a Coherent Verdi V5 continuous wave laser, emitting single-frequency light at $532 \mathrm{~nm}$ with an output power of up to $5 \mathrm{~W}$ and a bandwidth $<5 \mathrm{MHz}$. The laser's output frequency can either be tuned by modifying an intra-cavity etalon's temperature or by issuing control voltages onto two piezoelectric elements and thus altering the resonator's length. A control loop based on an High Finesse WSU 10 wavelengthmeter is installed to monitor and stabilize output frequencies with relative deviations below $2 \mathrm{MHz}$.

The output beam is directed via an articulated mirror arm (ILA GmbH) above the test rig, where it is formed into a light sheet of $45 \mathrm{~mm}$ width and $<0.5 \mathrm{~mm}$ thickness using an optical scanner arrangement (Röhle and Willert, 2001). Laser power is monitored by introducing a thin glass plate at the mirror arm exit and directing the deflected beam portion onto a photodiode behind a rotating diffusion disc. The light sheet illuminates Plane 41 through the downstream flange originally designed for five-hole probe traversing. For OP Iso only the area between NGV vanes 3 and 4 is covered by the light sheet, while for OPs OP150 and OP250 the sheet is traversed in lateral direction in order to illuminate the whole visible cross section.

Light scattered from the plane of interest is captured by a first lens and enters the transfer optics. In between two lenses in retro arrangement, a filter cell filled with molecular iodine as well as a bandpass filter (Barr, FWHM $1 \mathrm{~nm}$ ) are placed. Light transferred through the filter array is finally accumulated by a Hamamatsu C9100-13 EM-CCD camera. For OP Iso, the detection unit is placed at an angle of $90^{\circ}$ with respect to the plane of interest and, as indicated in the previous paragraph, thus observes the NGV pitch between vanes 3 and 4 . The spatial resolution for this setup is $4.7 \mathrm{pixel} / \mathrm{mm}$. In case of OPs OP150 and OP250, the spatial resolution is reduced and the detection unit is oriented at $79^{\circ}$ with respect to the measurement plane. Thus, two NGV pitches between vanes 2, 3 and 3,4 are visible in the camera's field of view. Methods given in Willert (2006) are used to calibrate the camera position as well as to dewarp the image data, finally yielding a spatial resolution of $2 \mathrm{pixel} / \mathrm{mm}$, which equals resolutions of $\sim 0.5 \mathrm{~mm}$ in radial and $\sim 0.15^{\circ}$ in tangential direction respectively.

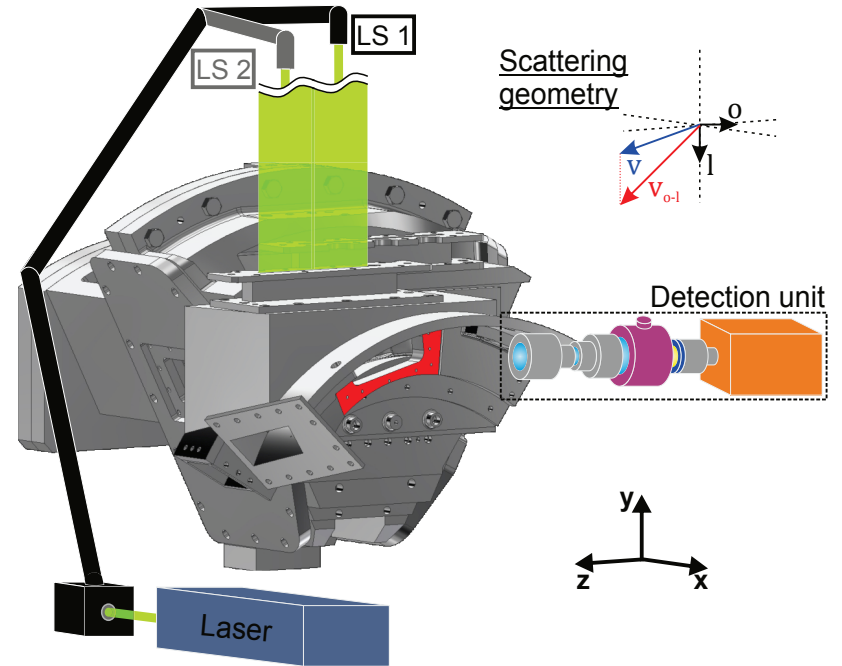

Fig. 3 Principle setup and optical arrangement: The laser beam is guided through an articulated mirror arm (black), formed into a light-sheet and enters the test rig from above along 1 . To cover the whole visible cross section, the sheet is traversed in lateral direction. Scattered light is captured along o by a first lens (grey) and enters the detection unit, where it is transferred through the iodine cell (pink) as well as a bandpass filter (blue) by two lenses in retro-arrangement (grey) and finally is imaged onto the camera (orange) sensor. The detected Doppler frequency-shift is proportional the projection of the flow velocity $\mathbf{v}$ onto $\mathbf{o}-\mathbf{l}\left(\mathbf{v}_{\mathrm{o}-1}\right)$.

\section{Results and discussion}

Velocity measurements by FSM-FRS rely on the optical Doppler frequency-shift

$\Delta \nu=\frac{\nu_{0}}{c}(\mathbf{o}-\mathbf{l}) \cdot \mathbf{v}$,

which is proportional to the projection of the threecomponent (3C) velocity vector $\mathbf{v}$ onto the bisector spanned by light-sheet $\mathbf{l}$ and observer direction $\mathbf{o}$. The expression incorporates the excitation frequency $\nu_{0}$ and the speed of light $c$. In order to reconstruct a $3 \mathrm{C}$ velocity field from FRS data, either light sheet orientation or observer position would have to be varied (Meyers and Komine, 1991; Doll et al, 2017b). In the current setup, only a single camera position as well as light-sheet direction could be realized. Thus, in the following, in order to compare both methods, five-hole probe $3 \mathrm{C}$ velocity data is converted to Doppler frequency-shifts according to the scattering geometry depicted in Fig. 3. Taking velocity uncertainties stated in Sect. 2.2 into account, this results in uncertainties of five-hole probe Doppler frequency-shifts of the order of $3 \mathrm{MHz}$. 
Table 2 Spatial averages of the difference $\overline{X_{i, \mathrm{~T}}-X_{i, \text { am }}}$ between results obtained using Tenti's (T) and the analytical lineshape model (am) for OPs Iso and OP150. Plus-minus signed values represent the spatial variation of the respective difference. $X_{i, \text { am }}$ and $\sigma_{X_{i}}$ denote spatial averages as well as absolute statistical uncertainties of FSM-FRS measurement results. For $O P 250$ the difference is calculated from spatial averages of Tenti and five-hole probe results.

\begin{tabular}{|c|c|c|c|c|c|c|c|c|c|}
\hline \multirow[b]{2}{*}{$X_{\hat{i}}$} & \multicolumn{2}{|c|}{ Iso } & \multirow[b]{2}{*}{$\sigma_{X_{\hat{i}}}$} & \multicolumn{2}{|c|}{ OP150 } & \multirow[b]{2}{*}{$\sigma_{X_{\hat{i}}}$} & \multicolumn{2}{|c|}{ OP250 } & \multirow[b]{2}{*}{$\sigma_{X_{\hat{i}}}$} \\
\hline & $\bar{X}_{\hat{i}, \mathrm{~T}}-X_{\hat{i}, \mathrm{am}}$ & $\overline{X_{\hat{i}, \mathrm{am}}}$ & & $\overline{X_{\hat{i}, \mathrm{~T}}-X_{\hat{i}, \mathrm{am}}}$ & $\overline{X_{\hat{i}, \mathrm{am}}}$ & & $\overline{X_{\hat{i}, \mathrm{~T}}}-\overline{X_{\hat{i}, \text { probe }}}$ & $\bar{X}_{\hat{i}, \text { probe }}$ & \\
\hline$p(\mathrm{hPa})$ & $-23 \pm 28$ & 840 & 56 & $-145.6 \pm 26$ & 1090 & 72 & -112 & 975 & 88 \\
\hline$T(\mathrm{~K})$ & $10.2 \pm 3$ & 285.9 & 2 & $10.1 \pm 2.6$ & 349.2 & 2.7 & 11.3 & 394.3 & 3.4 \\
\hline$\Delta \nu(\mathrm{MHz})$ & $-0.75 \pm 1.5$ & -18 & 1.7 & $-0.7 \pm 0.7$ & -157 & 1.9 & 6 & -219 & 2.7 \\
\hline
\end{tabular}

\subsection{Analysis of FSM-FRS accuracies}

Concerning the operating conditions given in Tab. 1, only OPs Iso and OP150 lie in the analytical lineshape model's calibrated region, whereas OP250 has to be evaluated using the standard lineshape model by Tenti et al (1974). To assess the error introduced by the standard model to OP250 measurement results, OPs Iso and OP150 are evaluated using Tenti's model as well. As flow conditions at Plane 41 of OPs OP150 and OP250 are expected to be comparable, resulting deviations between OP150's results obtained with the analytical lineshape model as well as Tenti's model will be a good indicator for the expected bias to OP250 results.

Results of the analysis are summarized in Tab. 2. In addition, statistical uncertainties to the measurands calculated using the methods given in Doll et al (2016) are provided as well. For OPs Iso and OP150, the offset introduced by the Rayleigh lineshape model is quantified by calculating the spatial average of the difference between results obtained using Tenti's as well as analytical lineshape model. The resulting average difference can be deemed representative for all sensor elements, which is indicated by spatial variations of the differences for pressure being well below and for temperature and Doppler frequency-shift being in the region of statistical uncertainty values. As for OP250 the analytical lineshape model cannot be applied, results obtained using Tenti's model are compared to five-hole probe data.

Concerning pressure results, the offset introduced in applying Tenti's lineshape model rises from $-23 \mathrm{hPa}$ for the isothermal operating condition to values above $-100 \mathrm{hPa}$ for OPs OP150 and OP250. This points to a possible relation between pressure offset due to the lineshape model and chamber pressure, which is $210 \mathrm{hPa}$ lower in case of OP Iso. Temperature deviations for all OPs are of the order $10 \mathrm{~K}$, whereas for Doppler frequency-shifts at OPs Iso and OP150, the difference between evaluations using Tenti's and analytical lineshape model are almost zero. This can be explained by the fact that a bulk motion of gas molecules inside the probe volume due to flow velocity shifts the complete
Rayleigh spectrum to higher or lower frequencies, but does not affect the Rayleigh scattering's spectral shape (Mielke et al, 2005). The offset in $\Delta \nu$ at OP250 between FSM-FRS results obtained using Tenti's model and five-hole probe data may be related to findings discussed in Sect. 3.2.

\subsection{Comparison of FSM-FRS and five-hole probe results}

The following comparison between five-hole probe and FSM-FRS measurements is based upon respective results obtained at operating point OP150. In Fig. 4, static pressure, temperature as well as Doppler frequency-shift maps measured with FSM-FRS and five-hole probe/temperature sensor are held against each other. As indicated in Sect. 2.2, the minimal wall distance feasible by the probe traverse is restricted by the probe head's dimensions, whereas FSM-FRS results further extend towards the upper and lower boundaries. In observing the plane of interest through optical access No. 3 (see Fig. 1), part of the flow channel, which can be accessed by the probe traverse, is obstructed due to geometrical restrictions and is thus limiting the camera's field of view. Overall, there is a general quantitative agreement for all shown quantities, whereas in terms of topology, certain discrepancies are visible.

Comparing static pressure maps of Fig. 4, both FSMFRS as well as five-hole probe results exhibit a similar absolute level of about $1100 \mathrm{hPa}$. A zone of heightened pressure between NGV vanes 2 and 3 is visible in the upper half of FSM-FRS results, which, although less pronounced and shifted in vertical direction, can also be recognized in five-hole probe data. A second zone of increased static pressure is visible in the center of FSM-FRS results, whereas, in contrast, five-hole probe pressure data exhibits a slight drop. As the region lies in the direct vicinity of $\mathrm{NGV}_{3}$, a possible reason for the discrepancies may lie in an insufficient correction of laser-induced background scattering. As the correction method introduced in Doll et al (2016) relies on interpolating a coarse grid of measured background light 

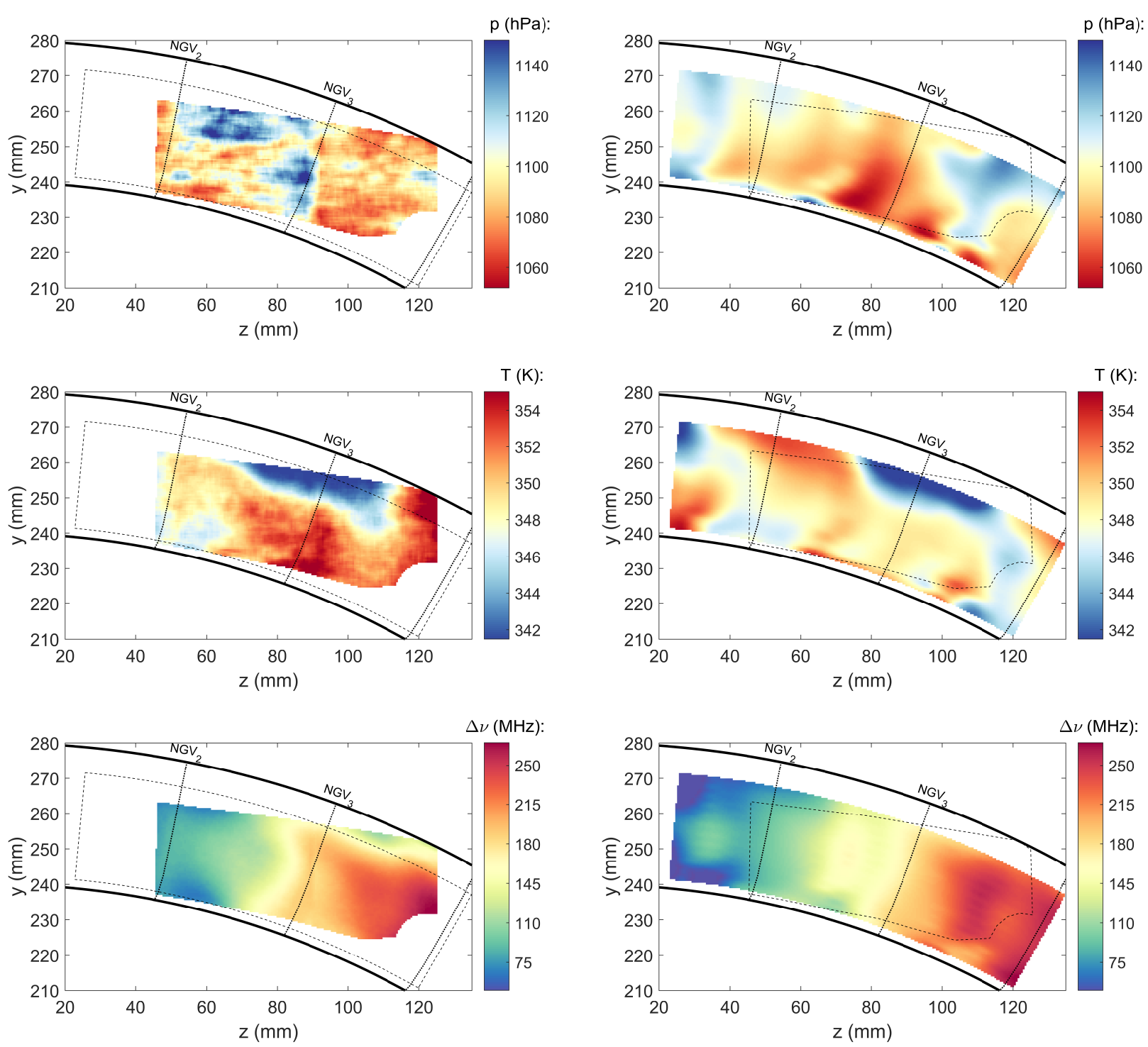

Fig. 4 Comparison of FSM-FRS (left) and five-hole probe (right) static pressure (top), temperature (middle) and Doppler frequency-shift (bottom) maps measured at OP150. Thick solid lines mark the upper (radius $280 \mathrm{~mm}$ ) and lower (radius $240 \mathrm{~mm}$ ) channel boundaries, dashed lines indicate areas investigated by the alternate method and dotted lines denote NGV trailing edge positions projected onto the measurement plane. Five-hole probe maps are interpolated from the probe grid to FSM-FRS results resolution. Results of both methods are smoothed with a moving average filter $(5 \times 5$ kernel size $)$.

onto the complete imaged region, structured elements such as NGV edges are not captured by the method, which results in incorrect background intensities and hence biased pressure readings for affected image areas. Concerning the NGV pitch between vanes 3 and 4 , FSM-FRS pressure results are almost constant in this region, whereas the five-hole probe map is governed by a streak of heightened static pressure values.

Temperature fields measured by FSM-FRS and temperature sensor show good agreement in terms of structure as well as absolute value. A cold streak, beginning in the middle of NGV pitch 2-3 and extending into the region between vanes 3 and 4 , is visible in both maps.
While the structure ends near the flow channel's centerline in FSM-FRS results, it persists until the lower bound of the probe traverse. Another cold spot near the lower end of $\mathrm{NGV}_{2}$ is visible in both data sets.

Regarding Doppler frequency-shifts measured by FSMFRS and calculated from three-component five-hole probe velocity data, both maps show good quantitative as well as qualitative agreement. The rise of absolute values from left to right is following the steady increase of $v_{\mathrm{y}}$ in lateral direction. Differences between both maps are most pronounced in the center near the trailing edge of $\mathrm{NGV}_{3}$, where a steep gradient is visible in FSM-FRS results, while five-hole probe data in this region appears 


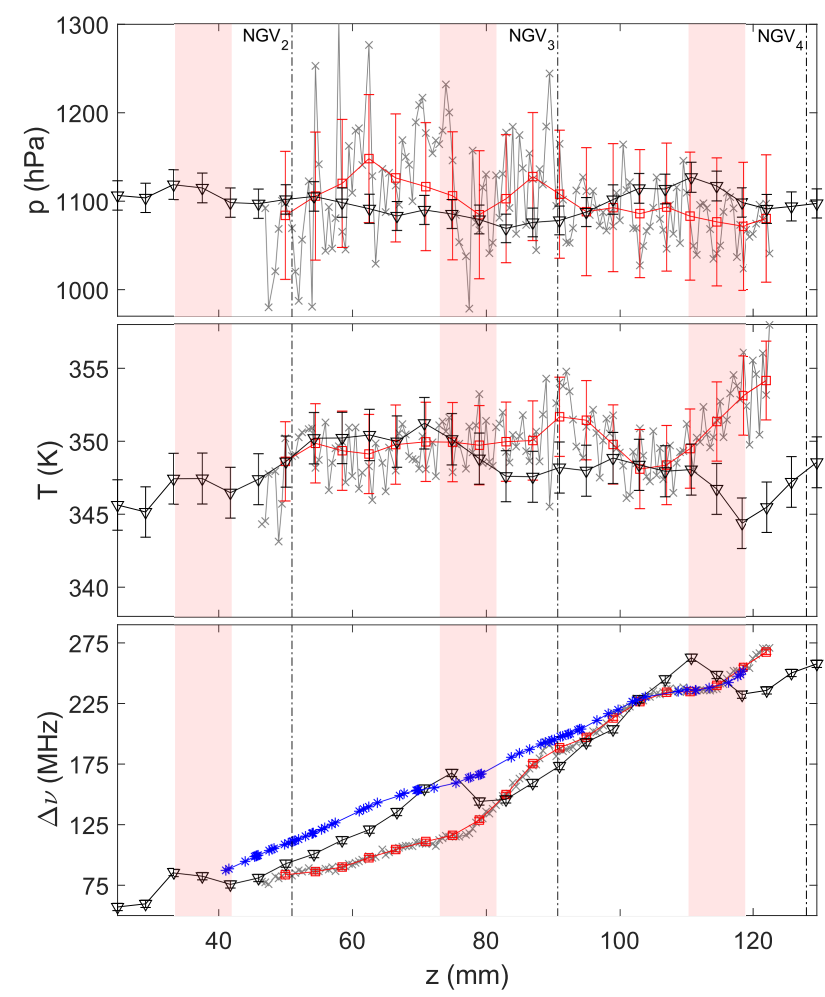

Fig. 5 Radial centerline profiles of pressure, temperature as well as Doppler frequency-shift at OP150 for FSM-FRS (gray, $\times$ ) and five-hole probe/temperature sensor (black, $\nabla$ ) measurement results. Red lines ( $\square$ ) represent FSM-FRS results mapped onto the five-hole probe measurement grid and resolution. Uncertainty values for five-hole probe/temperature sensor as stated in Sect. 2.2 and taken from Tab. 2 for FSM-FRS are marked by errorbars, respectively. Doppler frequency-shifts calculated from OP250 CFD velocity data are drawn in blue $(*)$. Dashed-dotted lines mark NGV trailing edge positions projected onto the measurement plane, red transparent areas indicate respective wake regions.

blurred as well as in the upper zone of NGV pitch 3-4, where in contrast to probe results, a gradient in radial direction is visible in FSM-FRS data.

In order to obtain further insights into the discrepancies between FSM-FRS and five-hole probe results, profiles of the measured quantities obtained near the flow channels' radial centerline are compared against each other in Fig. 5. Red transparent zones mark the wake regions corresponding to the respective NGV vane to the left. Wake positions and dimensions at Plane 41 are estimated using emperic formulas given in Lakshminarayana and Davino (1980) as well as Ainley and Mathieson (1951).

Due to the reduced pressure sensitivity caused by the normalization of signal intensities in Eq. (2), FSMFRS pressure results exhibit strong spatial variations, which are in accordance with the magnitude of statistical uncertainty visualized by respective errorbars. As indicated above in the comparison of FSM-FRS and five-hole probe pressure maps, besides a general agreement in magnitude, there is no strong correlation between both curves.

As observed for pressure results, the spatial variation of the depicted FSM-FRS temperature profile is well within the range of statistical uncertainty denoted by respective errorbars. The absolute level between both curves is in good agreement and similar structures are visible, however, the curves appear to be shifted from another in z-direction by an amount of $\sim 10 \mathrm{~mm}$. Besides any blocking effects from the probe on the temperature field, the displacement may be attributed to the differing measuring positions between temperature sensor and FSM-FRS light sheet. As already indicated in Sect. 2.2, the temperature sensor is fixed to the top of the probe shaft, which in consequence leads to a shift in $\mathrm{x}$ - and z-direction of the temperature sensor's actual measuring position. In addition, a slight re-adjustment of the laser sheet, which was necessary to compensate the test rig's movement while heating up, may have led to a positioning error of a few millimeters in FSM-FRS results.

The highest dynamics of all measured quantities can be observed in Doppler frequency-shifts, with values ranging from $75 \mathrm{MHz}$ up to $275 \mathrm{MHz}$ from the left to the right end of the investigated area. The FSM-FRS profile exhibits a steady rise, with a minor discontinuity visible at the trailing edge of $\mathrm{NGV}_{3}$. As mentioned in Sect. 2.3, the light sheet is traversed in z-direction so that the complete field of view is covered with laser light. The unavoidable movement of optical elements probably led to a slight misalignment of the l-vector and thus slightly affects the absolute level of measured frequency-shift values.

Doppler frequency-shifts calculated from five-hole probe velocity data and FSM-FRS results start at a similar absolute level next to $\mathrm{NGV}_{2}$ 's trailing edge, however, differences grow steady towards the adjacent wake region. While FSM-FRS results are not affected by the wake, the probe curve steeply drops towards the FSM-FRS profile. Starting to rise again at a lower slope, five-hole probe data crosses FSM-FRS frequency-shifts around $\mathrm{z}=100 \mathrm{~mm}$ and, again, drops below the FSMFRS curve when reaching the wake zone of $\mathrm{NGV}_{3}$, continuing at a lower absolute level.

Whereas FSM-FRS pressure data lack sufficient quality to draw detailed conclusions from the comparison and the bulk difference found between temperature distributions can be associated with differing measurement positions, Doppler frequency-shift curves calculated from five-hole probe velocities and FSM-FRS results clearly exhibit prominent dissimilarities, particularly inside the NGV airfoils' wake regions. In order to 
further clarify the wakes' influence on Doppler-frequency shift distributions, $3 \mathrm{C}$ velocity data extracted from CFD (computational fluid dynamics) calculations is transformed into Doppler frequency-shifts and compared to pressure probe and FSM-FRS results. The calculations are carried out at OP250 flow conditions applying the ANSYS Fluent solver with SAS (scale-adaptive simulation) turbulence modeling for a single-sector (instead of the experimental three-sector) configuration and periodic boundaries. The CFD domain covers both the combustor simulator and the NGV cascade. Further details on the numerical setup can be found in Cubeda et al (2018)

A radial centerline profile of respective CFD results is added to the comparison of experimentally obtained Doppler frequency-shifts in Fig. 5. Similar to five-hole probe and FSM-FRS results, the CFD curve is steadily growing from left to right at a comparable absolute level. However, neither of the airfoils' wake regions leads to a sudden drop of CFD Doppler frequency-shifts as it is observed in the five-hole probe data. Instead, CFD and FSM-FRS curves exhibit an upward sloping path in the wake region of $\mathrm{NGV}_{2}$. In-between the passage of $\mathrm{NGV}_{2}$ and $\mathrm{NGV}_{3}$ both profiles start to match in absolute value and slope, following a characteristic S-shape in the adjacent wake of $\mathrm{NGV}_{3}$. Persisting differences between CFD and FSM-FRS may be related to the differing operating point as well as the highly distorted inflow into the high-pressure NGV cascade, which is not entirely captured by the numerical setup.

Fig. 6 shows a comparison of velocity components acquired with the pneumatic probe and obtained from CFD calculations at the same radial centerline. Owing to the differing operating point, simulated velocities are on a higher absolute level compared to their measured counterparts. In-plane components $v_{y}$ and $v_{z}$ exhibit similar structures inside as well as outside the airfoils' wake regions. However, axial velocities measured by the five-hole probe are exhibiting local steep reductions, which are slightly shifted from the actual wake zones, followed by a steady growth until they reach an almost constant level in the passages. The CFD curve instead is following an oppositely pointing slope inside the corresponding wake regions. When projected onto the FSMFRS scattering geometry, these steep positive gradients in $v_{x}$ measured by the five-hole probe result in a sharp dropping of associated Doppler frequency-shifts. In conclusion with the findings of Fig. 5, these events can clearly be attributed to a gradient induced bias of the five-hole probe readings, introduced by strong pressure gradients present in the NGV airfoils' wake areas (see Sect. 1 and given references).

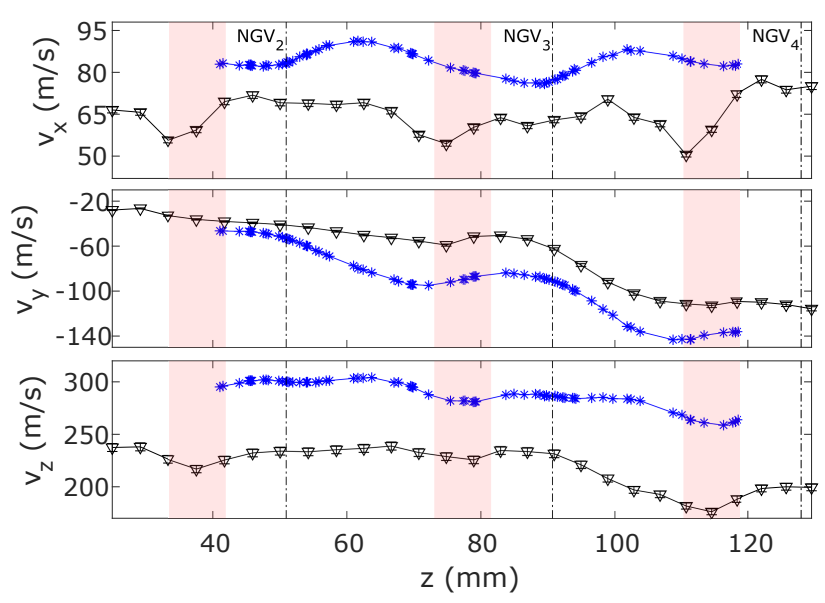

Fig. 6 Radial centerline profiles of velocity components $v_{\mathrm{x}}$, $v_{\mathrm{y}}, v_{\mathrm{z}}$ for five-hole probe measurements at OP150 (black, $\nabla$ ) and CFD calculations at OP250 (blue, *). Dashed-dotted lines mark NGV trailing edge positions projected onto the measurement plane, red transparent areas indicate respective wake regions.

\section{Conclusion}

The aero-thermal properties of the flow field downstream of an NGV cascade of a three-sector combustor simulator rig are charaterized by means of five-hole probe/temperature sensor measurements as well as laser-optical FSM-FRS diagnostics. The study discusses current capabilities as well as limitations of both methods when being applied to turbomachinery configurations.

FSM-FRS measurement accuracy strongly relies on the precise modeling of the Rayleigh scattering's spectral lineshape. With regard to operating points investigated herein, only OPs Iso and OP150 are found to lie within the calibrated region of an analytical lineshape model (Doll et al, 2016), while for OP250, the standard spectral modeling approach by Tenti et al (1974) has to be applied. In order to identify the erroneous impact of the lineshape model, OPs Iso and OP150 are evaluated using Tenti's model as well, yielding an average pressure offset of $-23 \mathrm{hPa}$ and $-145 \mathrm{hPa}$ as well as a temperature bias of $\sim 10 \mathrm{~K}$ for both OPs, respectively. Doppler frequency-shifts instead, as expected, remain almost unaffected by the lineshape modeling. When comparing FSM-FRS pressure and temperature results of OP250 to probe data, respective offsets of $-112 \mathrm{hPa}$ and $11.3 \mathrm{~K}$ are found to lie on a similar scale.

The direct comparison of five-hole probe/temperature sensor data and FSM-FRS measurement results for $O P 150$ reveals a good quantitative agreement between the methods, whereas in terms of flow topology, certain differences are evident. Concerning pressure, besides a general agreement in absolute level, no strong correlation is observable between flow structures in five-hole probe 
data and FSM-FRS results. Due to the lowered pressure sensitivity caused by the evaluation methodology applied here, the quality of FSM-FRS results is not sufficient to identify distinct attributes which could be related to the blockage of the flow passage by the probe body.

Regarding temperature, a lateral shift of about $10 \mathrm{~mm}$ between probe measurement and FSM-FRS results is identified, which can be attributed to the differing positions of the temperature sensor, being fixed to the top of the five-hole probe shaft and FSM-FRS measurement plane, which may be sightly shifted from the actual plane of interest after re-positioning the light sheet after heat-up. Besides, there is good quantitative as well as qualitative agreement between both methods, which implies only minor effects of probe blockage onto the temperature field.

Doppler frequency-shifts acquired by FSM-FRS and calculated from three-component velocity data measured by the five-hole probe are found to be on a similar absolute level. However, in the comparison of centerline profiles, both curves reveal distinct differences: While FSM-FRS results follow a continuous trend, the fivehole probe curve steeply drops inside the NGV airfoils' wake regions. To get further insight into the mechanisms causing these differences, a CFD solution calculated at OP250 is added to the comparison. The analysis reveals a bias to the axial velocity component measured by the five-hole probe, which can be attributed to strong pressure gradients present in the airfoils' wake areas. Besides this very distinct effect, passage flows in between two consecutive wake regions might be affected by the probe blockage. However, as CFD results are obtained at a differing operating point as well as numerical setup, presented results are ambiguous in this regard and cannot be used to draw reliable conclusions.

The FSM-FRS implementation presented herein has proven its capability to provide detailed insight into the complex aero-thermal flow topology dowstream of an NGV cascade with highly distorted inflow, representative for modern lean-burn combustion technology. With accuracies of $50-90 \mathrm{hPa}, 2-3 \mathrm{~K}$ and $2-3 \mathrm{MHz}$ in pressure, temperature and Doppler frequency-shift respectively and its superior spatial resolution, the FSM-FRS technique offers a viable alternative to invasive standard probe-based measuring approaches commonly applied in turbomachinery environments.

Acknowledgements The authors wish to to express their sincere thanks to Lorenzo Mazzei and Simone Cubeda for providing us with the CFD results presented in Sect. 3.2. We also would like to gratefully acknowledge the financial support by FACTOR (Full Aerothermal Combustor-Turbine interactiOns Research). FACTOR is a Collaborative Project co-funded by the European Commission within the Seventh Framework Program (2010-2016) under Grant Agreement No. 265985.

\section{References}

Ainley D, Mathieson G (1951) A Method of Performance Estimation for Axial-flow Turbines. Aeronautical Research Council. Reports and memoranda, H.M. Stationery Office, URL https://books.google.ch/books?id=G2WEuAAACAAJ

Aschenbruck J, Hauptmann T, Seume J (2015) Influence of a multi-hole pressure probe on the flow field in axial-turbines. In: 11th European Conference on Turbomachinery Fluid Dynamics and Thermodynamics, ETC 2015

Bacci T, Caciolli G, Facchini B, Tarchi L, Koupper C, Champion JL (2015) Flowfield and temperature profiles measurements on a combustor simulator dedicated to hot streaks generation. In: ASME Turbo Expo 2015: Turbine Technical Conference and Exposition, 56734, p V05CT17A001, DOI 10.1115/gt201542217

von der Bank R, Donnerhack S, Rae A, Cazalens M, Lundbladh A, Dietz M (2014) Lemcotec: Improving the core-engine thermal efficiency. In: ASME Turbo Expo 2014: Turbine Technical Conference and Exposition, American Society of Mechanical Engineers, pp V01AT01A001-V01AT01A001

Bryanston-Cross PJ, Towers CE, Judge TR, Towers DP, Harasgama SP, Hopwood ST (1992) The application of particle image velocimetry (piv) in a short-duration transonic annular turbine cascade. Journal of Turbomachinery 114(3):504-509, DOI $10.1115 / 1.2929173$

Chevrier M, Bertrand H (2017) Full Aero-thermal Combustor-Turbine interactiOn Research (FACTOR) - Final Publishable Summary Report. Tech. rep., FACTOR consortium

Cubeda S, Mazzei L, Bacci T, Andreini A (2018) Impact of predicted combustor outlet conditions on the aerothermal performance of film-cooled HPT vanes. In: ASME Turbo Expo 2015: Turbine Technical Conference and Exposition, 75921

Doll U, Stockhausen G, Willert C (2014) Endoscopic filtered Rayleigh scattering for the analysis of ducted gas flows. Experiments in Fluids 55(3):1-13

Doll U, Burow E, Stockhausen G, Willert C (2016) Methods to improve pressure, temperature and velocity accuracies of filtered Rayleigh scattering measurements in gaseous flows. Measurement Science and Technology 27(12):125,204 
Doll U, Stockhausen G, Heinze J, Meier U, Hassa C, Bagchi I (2017a) Temperature Measurements at the Outlet of a Lean Burn Single-Sector Combustor by Laser Optical Methods. Journal of Engineering for Gas Turbines and Power 139(2):021,507-021,507

Doll U, Stockhausen G, Willert C (2017b) Pressure, temperature, and three-component velocity fields by filtered rayleigh scattering velocimetry. Opt Lett 42(19):3773-3776

Fischer A, Büttner L, Czarske J, Gottschall M, Vogeler K, Mailach R (2012) Investigation of the tip clearance flow in a compressor cascade using a novel laser measurement technique with high temporal resolution. Journal of Turbomachinery 134(5):051,004-051,0049, DOI 10.1115/1.4004754

Fischer A, König J, Czarske J, Rakenius C, Schmid G, Schiffer HP (2013) Investigation of the tip leakage flow at turbine rotor blades with squealer cavity. Experiments in Fluids 54(2):1462, DOI 10.1007/s00348013-1462-1

Forkey J (1996) Development and demonstration of filtered rayleigh scattering: a laser based flow diagnostic for planar measurement of velocity, temperature and pressure. PhD thesis, Princeton University

Heinke W, König S, Matyschok B, Stoffel B, Fiala A, Heinig K (2004) Experimental investigations on steady wake effects in a high-lift turbine cascade. Experiments in Fluids 37(4):488-496, DOI 10.1007/s00348-004-0832-0

Hoenen HT, Kunte R, Waniczek P, Jeschke P (2012) Measuring failures and correction methods for pneumatic multi-hole probes. In: ASME Turbo Expo 2012: Turbine Technical Conference and Exposition, 44670, pp 721-729, DOI 10.1115/gt2012-68113

Lakshminarayana B, Davino R (1980) Mean velocity and decay characteristics of the guidevane and stator blade wake of an axial flow compressor. Journal of Engineering for Power 102(1):50-60, DOI $10.1115 / 1.3230231$

Luque S, Kanjirakkad V, Aslanidou I, Lubbock R, Rosic B, Uchida S (2015) A New Experimental Facility to Investigate Combustor-Turbine Interactions in Gas Turbines With Multiple Can Combustors. Journal of Engineering for Gas Turbines and Power 137(5), DOI $10.1115 / 1.4028714$

Meyers JF, Komine H (1991) Doppler global velocimetry-A new way to look at velocity. In: Laser Anemometry-Advances and Applications 1991, vol 1, pp 289-296

Mielke A, Seasholtz R, Elam K, Panda J (2005) Timeaverage measurement of velocity, density, temperature, and turbulence velocity fluctuations using rayleigh and mie scattering. Experiments in Fluids
39(2):441-454

Miles R, Lempert W (1990) Two-dimensional measurement of density, velocity, and temperature in turbulent high-speed air flows by UV Rayleigh scattering. Applied Physics B: Lasers and Optics 51:1-7

Miles R, Yalin A, Tang Z, Zaidi S, Forkey J (2001) Flow field imaging through sharp-edged atomic and molecular notch filters. Measurement Science and Technology 12(4):442, URL http://stacks.iop.org/0957$0233 / 12 / \mathrm{i}=4 / \mathrm{a}=308$

Qureshi I, Smith AD, Povey T (2012) Hp vane aerodynamics and heat transfer in the presence of aggressive inlet swirl. Journal of Turbomachinery 135(2):021,040-021,040-13, DOI 10.1115/1.4006610

Röhle I, Willert C (2001) Extension of Doppler global velocimetry to periodic flows. Measurement Science and Technology 12(4):420, URL http://stacks.iop.org/0957-0233/12/i=4/a=306

Sanders C, Terstegen M, Hölle M, Jeschke P, Schönenborn H, Fröbel T (2017) Numerical studies on the intrusive influence of a five-hole pressure probe in a high-speed axial compressor. In: ASME Turbo Expo 2017: Turbomachinery Technical Conference and Exposition, 50787, p V02AT39A009, DOI 10.1115/gt2017-63399

Schroll M, Doll U, G S, Meier U, Willert C, Hassa C, Bagchi I (2017) Flow Field Characterization at the Outlet of a Lean Burn Single-Sector Combustor by Laser-Optical Methods. Journal of Engineering for Gas Turbines and Power 139(1):011,503-011,503

Tenti G, Boley C, Desai R (1974) On the Kinetic Model Description of Rayleigh-Brillouin Scattering from Molecular Gases. Canadian Journal of Physics 52(4):285-290

Voges M, Willert CE, Mönig R, Müller MW, Schiffer HP (2012) The challenge of stereo PIV measurements in the tip gap of a transonic compressor rotor with casing treatment. Experiments in Fluids 52(3):581590, DOI 10.1007/s00348-011-1061-y

Wernet MP (2000) Development of digital particle imaging velocimetry for use in turbomachinery. Experiments in Fluids 28(2):97-115, DOI $10.1007 / \mathrm{s} 003480050015$

Willert C (2006) Assessment of camera models for use in planar velocimetry calibration. Experiments in Fluids 41(1):135-143, DOI 10.1007/s00348-006-01652

Woisetschläger J, Mayrhofer N, Hampel B, Lang H, Sanz W (2003) Laser-optical investigation of turbine wake flow. Experiments in Fluids 34(3):371-378, DOI 10.1007/s00348-002-0568-7 